\title{
Future Load Profiles Under Scenarios of Increasing Renewable Generation and Electric Transport
}

\author{
M. Allison*, E. Akakabota, G. Pillai, Member, IEEE \\ School of Science, Engineering and Design \\ Teesside University \\ Middlesbrough, UK \\ M.Allison@tees.ac.uk
}

\begin{abstract}
Load profiles are indispensable in the decision making process of power transmission and distribution companies. Increasing levels of customer-side renewable generation and electric transport will alter the nature of load profiles significantly. Traditional methods relying on historical data will not be suitable for modelling the increasingly complex power networks of the future. In this paper the feasibility of synthesising future load profiles under increasing levels of photovoltaic (PV) generation and electric vehicles $(\mathrm{EV})$ is investigated using an artificial neural network (ANN) based method, trained with publically available data. The performance of the proposed method is evaluated by using a case study developed for a targeted region in the UK. A comparison of results from the ANN model against those using Multiple Linear Regression (MLR) demonstrates the superior performance of ANN over MLR as well as proves the viability of ANN to synthesise future load profiles.
\end{abstract}

Keywords-load profiles; power networks; artificial neural networks, electric vehicle, photovoltaic

\section{INTRODUCTION}

Load profiles are essential for power transmission and distribution companies in order to make important decisions on the volume of generation, power purchase agreements, operation and maintenance scheduling, development of network infrastructure etc [1-2]. Traditionally load profiles are created using historical data [3], and are used in many countries to balance load demand [4]. In the near future, there will be a significant penetration of consumer-side generation using renewable technologies such as roof-top PV systems and electrical vehicle (EVs) in electricity distribution networks. This would cause massive changes to the nature of load profiles and hence it would not be possible to have true load profiles or close approximation based on historic load forecasting. This rising complexity of load profiles would be a major challenge for electrical power network operators. Hence it becomes important to develop suitable load profile generation methodologies that rely on publicly available data that aid different network related analyses by operators.

The exact penetration levels of consumer-side technologies such as PVs and EVs in the future energy demand mix is presently uncertain. The charging profiles of different EV technologies is also evolving as EV technology is evolving. Hence it is important to have a scalable method that can generate future load profiles under different PV and EV penetration levels. As there is a step change in load the objective is not to generate future load profiles based on historic datasets of load, but to use standardised load profiles and load/generation-weather relationships.

Previous literature reveals that a large proportion of the variability in electricity demand is dependent on weather variables such as air temperature, humidity, wind speed, cloud cover and irradiation [5-6]. It is also evident that the sensitivity of residential and commercial consumers electricity demand to meteorological variables is higher than that for industrial consumers [7]. Irradiance, air temperature, wind speed and air mass are weather features that affect PV power output [8]. Liu et al posit that there is no obvious correlation between wind speed and PV output power [9]. Aste et al find that performance ratio for crystalline silicon PV modules is fairly constant in the face of changes in air mass [10]. Seasonal variations in weather affect the PV output power from month to month. The existing literature seems to agree that irradiance and air temperature are the two most important weather features that impact on the output power of a PV system. The charging profiles of EVs have no obvious correlation to weather [11]. They are dictated by consumer driving behaviour which in turn is correlated to the socio-economic factors of the region. As temperature and irradiance are influencing parameters common to load and PV generation, it makes sense to include these as inputs for generating future aggregate load profiles.

In this paper the feasibility of using publicly available weather and electrical vehicle charging data to generate future penetration level scenario based residential load profiles is investigated. An artificial neural network (ANN) based method is proposed to synthesize future residential load profiles based on photovoltaics (PV) and electrical vehicle (EV) penetration levels and weather data as the input. The main contribution of this paper is the development of a computational procedure for PV and EV penetration scenario-based future load profile generation based on public data. The procedure developed is tested for a case study in Middlesbrough, UK.

The rest of the paper is organized as follows: Data inputs for this study, PV generation modelling and ANN based load profile generation methodology are described in Section II; Simulation results are presented and discussed in Section III and Conclusions are drawn in Section IV. 


\section{DATA AND MeThodology}

\section{A. Data Description}

The UK Energy Research Centre (UKERC) has developed load profile models for all 4 seasons of a typical year [12]. The load data used in this study is for residential customers unrestricted by usage timings. The load profiles from UKERC have hourly time-resolution and are publicly available. As described in the previous section, temperature and irradiation are the main weather data to be considered. There are a number of weather databases which provide weather data for a typical year for different locations such as NREL (National Renewable Energy Laboratory) National Solar Radiation Data base, NASA Surface meteorology and Solar Energy, PVGIS (Photovoltaic Geographical Information System) climate-SAF etc. PVGIS climate-SAF was selected as the reference solar database for the UK as it provides up-to-date data in the public domain for Europe. The data is available with hourly time resolution for 365 days a year. A MATLAB program was written to create seasonal average hourly weather (temperature and global irradiation) datasets. The Low Carbon London (LCL) project conducted customer trials of new transport and heating loads on distribution networks in London. Residential EV charging profile data for this paper is taken from the project [13].

\section{B. PV Generation}

Middlesbrough, UK is considered as the region where future load profiles are to be generated. In the UK, PV systems of $4 \mathrm{kWp}$ rating are eligible for the highest feed-in tariff incentive. Hence this system size was considered. The crystalline silicon PV technology was selected as it is the most mature PV technology with the highest market share. Typical PV systems were modelled using PVGIS 5 online software which generates PV output data with hourly time resolution for 365 days a year. Seasonal average typical PV generation datasets were created using a MATLAB program similar to that described for weather data. Fig.1 shows the seasonal variation in PV generation. As expected summer and spring months have higher power outputs for longer duration as compared to autumn and winter.

\section{C. $P V$ and EV Penetration Scenarios}

$\mathrm{PV}$ and EV penetration level in this work is defined as the ratio of the number of houses with a typical PV system or EV to the total number of houses in the distribution network for which the load profile is representative. In this work, PV penetration level was varied in steps of $10 \%$ from 0 to $100 \%$, along with a similar variation in $\mathrm{EV}$ penetration level corresponding to PV penetration level.

\section{Creation of composite future load profiles}

Composite future load profiles are essential for testing the feasibility of the ANN based load profile generation methodology. After generating seasonal PV generation profiles, composite future load profiles, for the whole range of $\mathrm{EV}$ and PV penetration scenarios described in the previous section for all seasons of a typical year, were created. This was done by aggregating seasonal UKERC profile class 1 load profiles with penetration-level-weighed PV generation (negative demand) profiles and EV charging profiles from LCL. As future load profiles for the penetration scenarios described are not yet available these composite load profiles were construed as close substitutes to the actual.

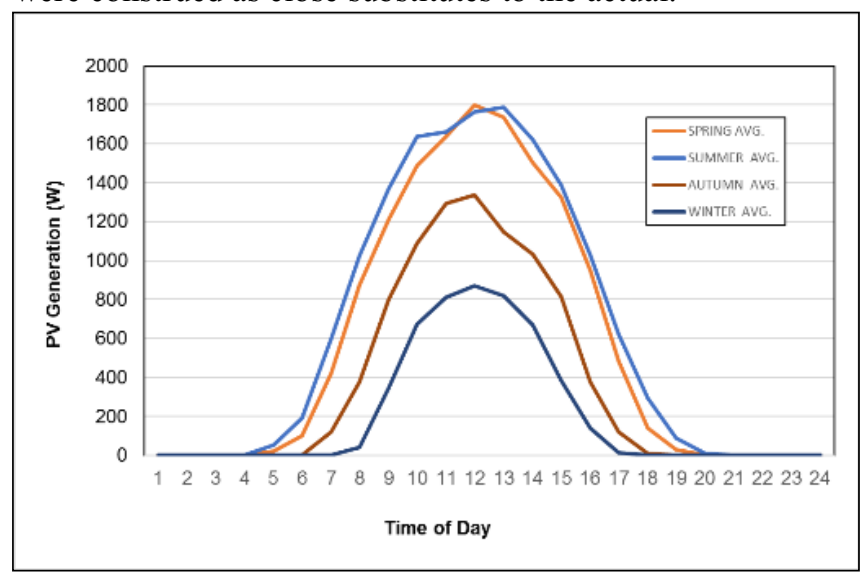

Fig. 1. Seasonal variation in PV output profiles of the typical PV system.

\section{E. Formulation of the prediction model}

Fig. 2 shows the block diagram of the computational procedure for formulation of the $\mathrm{PV}$ and $\mathrm{EV}$ penetration scenario-based future load profile prediction model.

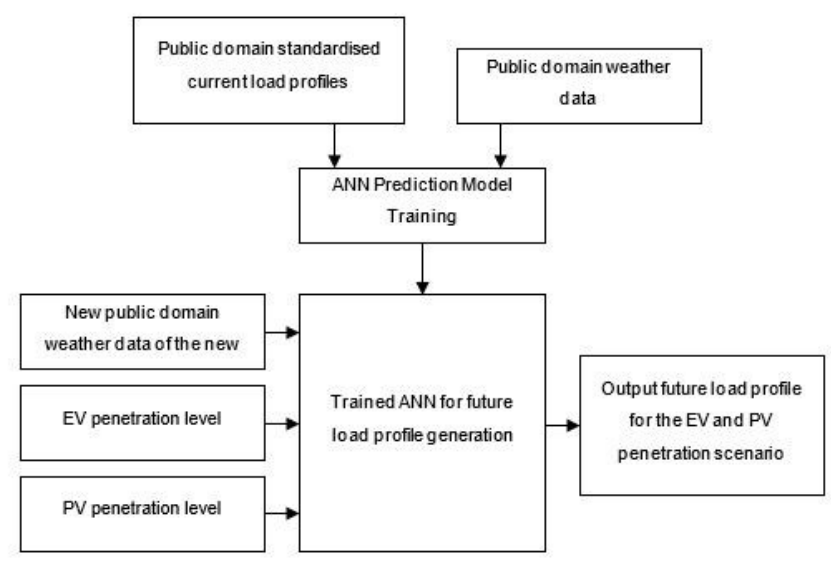

Fig. 2. Proposed ANN future load profile prediction model.

As described in the introduction section load profiles and PV generation are correlated to temperature and irradiance. Future load profiles are required for different PV and EV penetration levels. The model developed should be able to predict future load profiles under different weather and $\mathrm{PV} / \mathrm{EV}$ scenarios. For ease of reproduction and to aid use by electric network operators the data requirements of the method should be met from public domain data. ANN method was chosen for prediction because of its advantages such as its ability to identify and capture complex nonlinear relationships between dependent and independent variables, less formal statistical training requirement, ability to recognise all potential interactions between predictor variables and the availability of a range of different training algorithms as part of a MATLAB toolbox [14]. To visualise the complexity of the ANN training and prediction requirements, a similar prediction is attempted using Multiple Linear Regression (MLR). MLR is a prediction method that attempts to model the relationship between two or 
more input variables and a target output variable by fitting a linear equation between the input and output.

\section{F. ANN Design and Training}

ANNs are universal function approximators capable of mapping any nonlinear function. In terms of computational structure, ANNs are composed of neurons, which at a very basic level mimic neurons in the human body in terms of learning and processing information. In this work, a feed forward neural network is used. In this ANN design, neurons are arranged in successive layers and information flows from the input layer to the hidden layer and then to output layer. The method used for ANN training is supervised learning where the training data includes both the input and the target outputs. Levenberg-Marquardt algorithm is used for ANN training owing to its training speed and ease of implementation using MATLAB neural network toolbox.

The input variables for the ANN model was time of day, global irradiation $\left(\mathrm{W} / \mathrm{m}^{2}\right)$, temperature $\left({ }^{\circ} \mathrm{C}\right)$, PV penetration level $(\%)$ and $\mathrm{EV}$ penetration level (\%). The output is load for the particular hour $(\mathrm{kW})$. The ANN model was trained on input weather and target load data for spring, summer and winter for the range of penetration scenarios described in section II.C. As complexity increases the difficulty in training and the training time, it was aimed to keep the ANN structure as simple as possible. A single hidden layer was considered between the input and output layers. Initially the ANN was trained with 5 input nodes corresponding to the 5 input variable, 10 hidden nodes (default MATLAB architecture) and 1 output node corresponding to the load. The use of the default ANN architecture gave a Pearson correlation coefficient $(R)$ value close to 1 , between predicted outputs and targets outputs of the ANN. The ANN was re-trained after reducing the number of hidden nodes by one to see the decline in R-value. This iterative procedure continued until the optimum ANN architecture with 6 hidden nodes shown in Fig. 3 resulted. The feasibility of the proposed method for future load profile generation was investigated using the case study of different PV and EV penetrations for the autumn season for which the ANN does not have a priori knowledge from training.

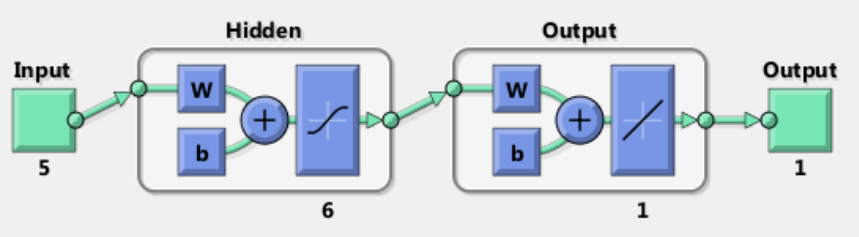

Fig. 3. ANN architecture for future load profile prediction model.

\section{G. Prediction performance metrics}

The statistical metrics used for examining the prediction accuracy and comparing the performance of ANN to regression were: the root mean square error (RMSE), the mean absolute error (MAE) and the mean absolute percentage error (MAPE). They are defined by the following statistical equations:

$$
R M S E=\sqrt{\frac{\sum_{i=1}^{n}\left(P_{i}-A_{\bar{L}}\right)^{2}}{n}}
$$

$$
\begin{aligned}
& M A E=\frac{\sum_{i=1}^{n} \mid\left(P_{i}-A_{i}\right)}{n} \\
& M A P E=\frac{\sum_{i=1}^{n}\left|\frac{\left.\mid P_{i}-A_{i}\right)}{A_{i}}\right|}{n} \times 100
\end{aligned}
$$

Where $\mathrm{P}_{\mathrm{i}}$ and $\mathrm{A}_{\mathrm{i}}$ are the synthetic load profile data and actual load profile data at the $i^{\text {th }}$ point respectively, and $n$ is the total number of data points (i.e. 24 per load profile for hourly resolution).

\section{RESULTS AND DISCUSSION}

To validate the suitability of the proposed ANN for generating synthetic future residential load profiles, the performance of ANN model was compared to multiple linear regression (MLR) - a common prediction model. This section compares the training and prediction performance of both models.

\section{A. Training}

Both ANN and regression models were trained using the same input weather and target load data, and the whole range of penetration levels of PV and EV described in section II.C, with hourly resolution. The training data was for spring, summer and winter of the typical year. To analyse the fitness of the model the output of the ANN and regression models with the training data was compared to the actual load profiles (targeted training outputs). Owing to the 112 (in-total) combinations of $\mathrm{PV}$ and $\mathrm{EV}$ penetration scenarios and 3 seasons, there were 336 twenty-four hour load profiles (ANN, MLR and actual) to be compared. Fig. 4 and 5 show representative comparison obtained for 2 (out of the 300 scenarios) namely: $10 \%$ PV penetration and $10 \% \quad \mathrm{EV}$ penetration in spring and 50\% PV penetration and 70\% $\mathrm{EV}$ penetration in winter.

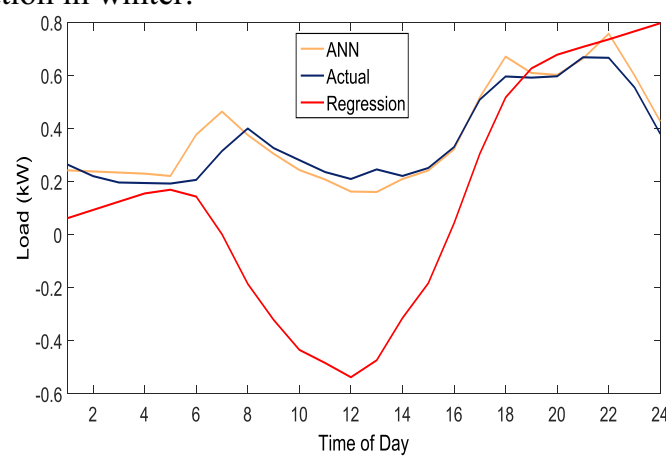

Fig. 4. Training results for $10 \% \mathrm{PV}$ and $10 \% \mathrm{EV}$ penetration in spring

For a typical day in spring, with $10 \% \mathrm{PV}$ penetration and $10 \%$ EV penetration, ANN shows a markedly better approximation to the actual load profile than MLR - as shown in Fig. 4. In Fig. 5, for the scenario of 50\% PV penetration and $70 \% \mathrm{EV}$ penetration on a typical winter, the difference between the training performance of both models is not so apparent. However, because these two scenarios are only small portions of a large training dataset, a statistical description of training performance over the entire dataset is desirable and is described by the MAPE, MAE and RMSE values in Table 1. 


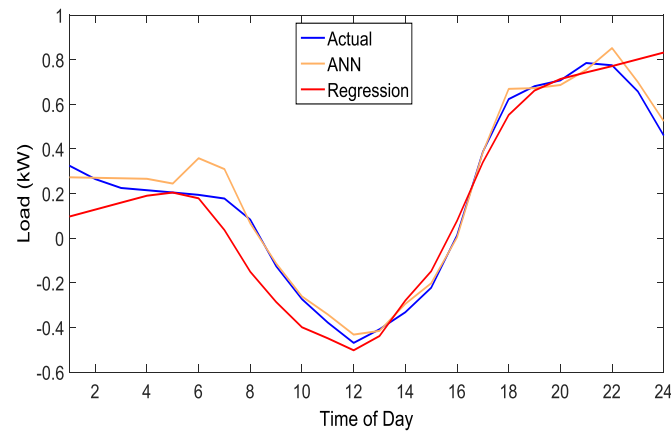

Fig. 5. Training results for $50 \% \mathrm{PV}$ and $70 \% \mathrm{EV}$ penetration in winter

TABLE I.

TRAINING PERFORMANCE OF ANN AND MLR FOR SPRING, SUMMER AND WINTER UNDER VARIOUS PV AND EV PENETRATION SCENARIOS

\begin{tabular}{|r|c|c|}
\hline & ANN & MLR \\
\hline MAPE & $7.2931 \%$ & $18.3632 \%$ \\
\hline MAE & 0.0349 & 0.1959 \\
\hline RMSE & 0.0492 & 0.2562 \\
\hline
\end{tabular}

From Table 1, it is apparent that ANN has much better training accuracy than MLR. MAPE of $7.29 \%$ for ANN means that the ANN trains with about $93 \%$ accuracy as compared to about $82 \%$ for MLR. The MAE and RMSE values also support the fact that ANN trains better.

\section{B. Validation}

Input weather data, PV and EV penetration levels and composite load profiles for autumn season of the typical year (for which the ANN models have no a priori knowledge) was used to test both ANN and MLR prediction models. Fig. 6 compares the predicted load profile for a day in autumn with the actual load profile, for a scenario of $20 \% \mathrm{PV}$ penetration and $30 \% \mathrm{EV}$ penetration. The predicted load profile using ANN closely approximates the actual load profile. On the other hand, MLR prediction shows a marked divergence from the actual load profile. The superior prediction performance of ANN is statistically supported by Table 2, with MLR showing a prediction accuracy of just about $15 \%$ for the test season (autumn). The ANN model has been proved to be a viable model for generation of synthetic load profile in the face of increasing penetration of $\mathrm{PV}$ and EV resources. The complexity of the prediction process can be easily visualised from the comparison to the MLR model.

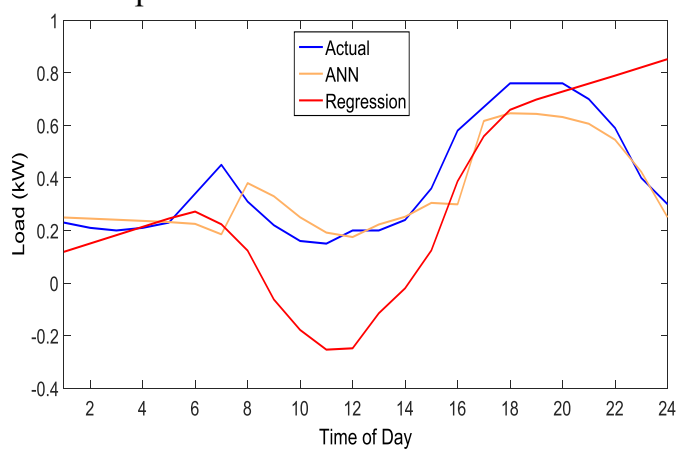

Fig. 6. Testing results for $20 \% \mathrm{PV}$ and $30 \% \mathrm{EV}$ penetration in autumn
TABLE II.

TESTING PERFORMANCE OF ANN AND MLR FOR 20\% PV PENETRATION AND $30 \%$ EV PENETRATION IN AUTUMN

\begin{tabular}{|r|c|c|}
\hline & ANN & MLR \\
\hline MAPE & $20.8407 \%$ & $74.8303 \%$ \\
\hline MAE & 0.0765 & 0.1959 \\
\hline RMSE & 0.1035 & 0.2481 \\
\hline
\end{tabular}

\section{CONCLUSIONS}

The feasibility of using an ANN based method to synthesise future residential load profiles under increasing levels of EV/PV penetration was investigated in this paper. The performance of the ANN model was evaluated through the use of a case study developed for a targeted region of the UK.

In order to minimise training difficulty and time the structure of the ANN model was kept as simple as possible. The model was trained using the Levenberg-Marquardt algorithm with publically available data.

The performance of the ANN model was compared to a MLR model which had been trained using the same data in a case study of different EV and PV penetration levels. Statistical analysis of the experimental results showed that during testing the ANN model performed with an accuracy of approximately $79 \%$ compared to the MLR model accuracy of approximately $15 \%$. The experimental results show that the ANN model has the ability to capture non-linear relationships even when trained with limited data from publically available sources.

The results prove the feasibility of the proposed ANN based method for synthesising future residential load profiles under increasing levels of EV and PV penetration. The computational method presented in this paper could be used to replace traditional methods which are not suitable for modelling the increasingly complex power networks of the future.

This work will be extended to other renewables and customer classes in future.

\section{REFERENCES}

[1] S. Fan, L. Chen, and W. J. Lee, "Short-term load forecasting using comprehensive combination based on multi-meteorological information," in Proc. IEEE Ind. Commercial Power Syst. Tech. Conf., May 4-8, 2008, pp. 1-7.

[2] A. Ahmad, N. Javaid, M. Guizani, N. Alrajeh and Z. A. Khan, "An Accurate and Fast Converging Short-Term Load Forecasting Model for Industrial Applications in a Smart Grid," in IEEE Transactions on Industrial Informatics, vol. 13, no. 5, pp. 2587-2596, Oct. 2017.

[3] B. V. M. Vasudevarao, M. Stifter and P. Zehetbauer, "Methodology for creating composite standard load profiles based on real load profile analysis," 2016 IEEE PES Innovative Smart Grid Technologies Conference Europe (ISGT-Europe), Ljubljana, 2016, pp. 1-6.

[4] A. Rahman and S. I. Chowdhury Arnob, "Developing load profile for domestic customers of Dhaka city through statistical prediction," 2016 3rd International Conference on Electrical Engineering and Information Communication Technology (ICEEICT), Dhaka, 2016, pp. 1-6.

[5] C. Wang, G. Grozev and S. Seo, "Decomposition and statistical analysis for regional electricity demand forecasting", Energy, vol. 41, no. 1, pp. 313-325, 2012. 
[6] M. Thatcher, "Modelling changes to electricity demand load duration curves as a consequence of predicted climate change for Australia", Energy, vol. 32, no. 9, pp. 1647-1659, 2007.

[7] F. Apadula, A. Bassini, A. Elli and S. Scapin, "Relationships between meteorological variables and monthly electricity demand", Applied Energy, vol. 98, pp. 346-356, 2012.

[8] T. Huld and A. Amillo, "Estimating PV Module Performance over Large Geographical Regions: The Role of Irradiance, Air Temperature, Wind Speed and Solar Spectrum", Energies, vol. 8, pp. 5159-5181, 2015.

[9] F. Liu, R. Li, Y. Li, R. Yan and T. Saha, "Takagi-Sugeno fuzzy modelbased approach considering multiple weather factors for the photovoltaic power short-term forecasting", IET Renewable Power Generation, vol. 11, no. 10, pp. 1281-1287, 2017.

[10] N. Aste, C. Del Pero and F. Leonforte, "PV technologies performance comparison in temperate climates", Solar Energy, vol. 109, 2014.

[11] J. Brady and M. O'Mahony, "Modelling charging profiles of electric vehicles based on real-world electric vehicle charging data", Sustainable Cities and Society, vol. 26, pp. 203-216, 2016.

[12] "UKERC Energy Data Centre", Data.ukedc.rl.ac.uk. [Online]. Available: http://data.ukedc.rl.ac.uk/cgi-bin/dataset_catalogue/view.cgi.py?id=6. [Accessed: 05- Nov- 2017].

[13] "Impact of Electric Vehicle and Heat Pump loads on network demand profiles", UK Power Networks, 2014.

[14] J. Tu, "Advantages and disadvantages of using artificial neural networks versus logistic regression for predicting medical outcomes", Journal of Clinical Epidemiology, vol. 49, no. 11, pp. 1225-1231, 1996. 